\title{
FRÉCHET ALGEBRAS GENERATED BY CERTAIN OF THEIR ELEMENTS
}

\author{
S. OUZOMGI \\ Department of M.thematics \\ The Pennsylvania State University \\ Abington, PA 19001

\section{REDLIN} \\ Depaitment of Mathematics \\ The Pennsylvania State University \\ Abington, PA 19001

\section{S. WATSON} \\ Department of Mathematics \\ California State University \\ Long Beach, CA 90840
}

(Received April 26, 1993 and in revised form August 2, 1993)

\begin{abstract}
We consider $F$-algebras $A$ that are generated by elements of the form $z,\left(z-\lambda_{1} e\right)^{-1}$, $\ldots,\left(z-\lambda_{N} e\right)^{-1}$, where $e$ is the identity. If $A$ has no topclogical divisors of zero we show that $A$ is isomorphic to $H(\Omega)$, where $\Omega$ is a finitely connected region. We also study $F$-algebras in which $\left\{e, z, z^{-1}, z^{2}, z^{-2}, \ldots\right\}$ is a basis.

1991 Mathematics Subject Classification. 46J35

Key words and phrases. Fréchet algebras, topological divisors of zero, algebras of holomorphic functions, Runge's theorem.
\end{abstract}

\section{INTRODUCTION}

The algebra $H(\Omega)$ of holomorphic functions on a region $\Omega$, with the compact-open topology has been characterized among $F$-algebras in several ways. Rudin [1] proved that a uniform $F$ algebra which satisfies a form of the maximum modulus principle is an algebra of holomorphic functions. Birtel [2] showed that under certain conditions a singly-generated $F$-algebra is the algebra of entire functions, and under other conditions it is shown in [3] that such an algebra is the algebra of holomorphic functions on a simply connected domain. Meyers [4] characterized $H(\Omega)$ by using the property that bounded sets are relatively compact. Carpenter [5] used the existence of derivations to characterize $H(\Omega)$. Arens [6] gave conditions on a singly-rationallygenerated $F$-algebra which ensure that the algebra is the direct sum of its radical and an algebra of holomorphic functions on a region. Brooks [7] showed that the same conclusion holds for locally $m$-convex algebras in which a certain boundary is empty. In [8] and [9] conditions are given on an $F$-algebra generated by a finite number of elements that guarantee that the algebra is an algebra of holomorphic functions. These and other $F$-algebra characterizations of $H(\Omega)$ are in terms of well known properties of $H(\Omega)$, such as Liouville's theorem [2], the maximum modulus principle [1], Montel's theorem [4], the Cauchy estimate [6], Taylor's theorem [9], the existence of certain derivations [5], and others.

In this paper we consider $F$-algebras $A$ that are generated by certain of their elements, namely

$$
z,\left(z-\lambda_{1} e\right)^{-1}, \ldots,\left(z-\lambda_{N} e\right)^{-1}
$$

where $e$ is the identity of $A$ and $\lambda_{1}, \ldots, \lambda_{N} \in \mathbb{C}$. Under one additional assumption, that $A$ has no topological divisors of zero, we show that $A$ is isomorphic to $H(\Omega)$, where $\Omega$ is a finitely 
connected region. This can be viewed as a characterization of $H(\Omega)$ in terms of Runge's theorem. We also consider $F$-algebras in which $\left\{e, z, z^{-1}, z^{2}, z^{-2}, \ldots\right\}$ is a basis for $A$.

An $F$-algebra is a complete metrizable locally $m$-convex algebra over $\mathbb{C}$. (We consider only commutative $F$-algebras.) The topology of an $F$-algebra $A$ is given by an increasing sequence of seminorms $\left\{p_{n}: n \in \mathbb{N}\right\}$, and $A$ is isomorphic to an inverse $\operatorname{limit} \underset{\leftarrow}{\lim }\left(A_{n}, \pi_{n m}\right)$ of Banach algebras as follows: $A_{n}$ is the completion of $A / \operatorname{ker}\left(p_{n}\right)$ in the induced norm, and for $n \leq m$ the map $\pi_{n m}: A_{m} \rightarrow A_{n}$ is the natural homomorphism. The maximal ideal space $\mathcal{M}(A)$ consists of all non-zero continuous multiplicative linear functionals on $A$ endowed with the weak topology generated by the Gelfand transforms $\widehat{x}: \mathcal{M}(A) \rightarrow \mathbb{C}$, where $\widehat{x}(f)=f(x)$. The algebra of all Gelfand transforms is $\widehat{A}=\{\widehat{x}: x \in A\}$ equipped with the compact-open topology. The Gelfand map $\Gamma: A \rightarrow \widehat{A}$ is continuous and is a bijection if $A$ is semisimple. The quotient map $\pi_{n}: A \rightarrow A / \operatorname{ker}\left(p_{n}\right)$ induces a homeomorphism of the maximal ideal space $\mathcal{M}\left(A_{n}\right)$ onto a compact subset $M_{n}$ of $\mathcal{M}(A)$. For $n \leq m$ we have $M_{n} \subset M_{m}$ and $\mathcal{H}(A)=\cup M_{n}$. The joint spectrum of $\left\{w_{1}, w_{2}, \ldots, w_{N}\right\} \subset A$ is the set

$$
\sigma\left(w_{1}, w_{2}, \ldots, w_{N}\right)=\left\{\left(f\left(w_{1}\right), f\left(w_{2}\right), \ldots, f\left(w_{N}\right)\right): f \in \mathcal{M}(A)\right\}
$$

The joint spectrum of these elements in $A_{n}$ is

$$
\sigma_{n}\left(w_{1}, w_{2}, \ldots, w_{N}\right)=\left\{\left(f\left(w_{1}\right), f\left(w_{2}\right), \ldots, f\left(w_{N}\right)\right): f \in M_{n}\right\}
$$

We have $\sigma=\bigcup \sigma_{n}$. The elements $\left\{w_{1}, w_{2}, \ldots, w_{N}\right\}$ generate $A$ if $A$ is the smallest closed subalgebra containing these elements and the identity $e$. In this case the spectrum map

$$
\begin{gathered}
\varphi: \mathcal{H}(A) \rightarrow \sigma\left(w_{1}, w_{2}, \ldots, w_{N}\right) \\
: f \mapsto\left(f\left(w_{1}\right), f\left(w_{2}\right), \ldots, f\left(w_{N}\right)\right)
\end{gathered}
$$

is a continuous bijection [7].

An element $z$ in a Banach algebra $B$ is a topological divisor of zero if the map $T_{z}: B \rightarrow z B$ is not an isomorphism. In an $F$-algebra $A, z$ is a topological divisor of zero if for each sequence $\left\{p_{n}: n \in \mathbb{N}\right\}$ of seminorms defining the topology of $A$ there exists a $k$ such that $\pi_{k}(z)$ is a topological divisor of zero in the Banach algebra $A_{k}$ (See Michael [10]). When we say that an algebra has no topological divisors of zero, we will mean, of course, that it has no nonzero ones.

\section{A CHARACTERIZATION OF RUNGE ALGEBRAS}

If $\Omega \subset \mathbb{C}$ is a finitely connected domain, and $H(\Omega)$ is endowed with the compact-open topology, then by Runge's Theorem, $H(\Omega)$ is generated by rational functions with poles in $\mathbb{C}_{\infty} \mid \Omega$. In fact, if $\lambda_{1}, \ldots, \lambda_{N}$ are points chosen one from each component of $\mathbb{C}_{\infty} \mid \Omega$, then the rational functions with poles at $\lambda_{1}, \ldots, \lambda_{N}$ suffice to generate $H(\Omega)[11$, page 200]. It is also well-known that $H(\Omega)$ has no topological divisors of zero. We will show that these two properties characterize $H(\Omega)$ as an $F$-algebra.

An $F$-algebra is a Runge algebra if there is an element $z \in A$ and complex numbers $\lambda_{1}, \ldots, \lambda_{N}$ such that $A$ is generated by $\left\{z, w_{1}, \ldots, w_{N}\right\}$, where $w_{1}=\left(z-\lambda_{1} e\right)^{-1}, \ldots$, $w_{N}=\left(z-\lambda_{N} e\right)^{-1}$ 
PROPOSITION 2.1. If $A$ is a Runge algebra weth no topological divisors of zero, then $\sigma(z)$ is a finitely connected domain.

Proof. If $\sigma(z)$ were to contain a boundary point $\alpha$, then $z-\alpha e$ would be a topological divisor of zero [10, Proposition 11.8]. Thus $\sigma(z)$ is open. Now suppose $\sigma(z)$ is the disjoint union of nonempty open sets $S$ and $T$, and let $\chi_{S}$ and $\chi_{T}$ be their characteristic functions. These are analytic on $\sigma(z)$, so by the functional calculus [12] we can find $x, y \in A$ such that $\widehat{x}=\chi_{S} \circ \widehat{z}$ and $\widehat{y}=\chi_{T} \circ \widehat{z}$; hence $\widehat{x y}=0$. Since $A$ has no topological divisors of zero, it follows that $A$ is semisimple and so $x y=0$. But then $x$ and $y$ would be proper zero-divisors, a contradiction. Thus $\sigma(z)$ is connected, and so it is a domain.

Let $\varphi: \mathcal{H}(A) \rightarrow \sigma\left(z, w_{1}, \ldots, w_{N}\right)$ be the spectrum map and let

$$
\psi: \sigma\left(z, w_{1}, \ldots, w_{N}\right) \rightarrow \sigma(z)
$$

be the map taking $\left(f(z), f\left(w_{1}\right), \ldots, f\left(w_{N}\right)\right)$ to $f(z)$. Clearly $\psi$ is a continuous bijection, and hence so is $\varphi_{0}=\psi \circ \varphi$. For $t \in \sigma(z)$ let $f_{t}=\varphi_{0}^{-1}(t)$, and for $x \in A$ define $\widetilde{x}: \sigma(z) \rightarrow \mathbb{C}$ to be the map taking $t$ to $\widetilde{x}(t)=\widehat{x}\left(f_{t}\right)=f_{t}(x)$, as indicated in the commutative diagram below.

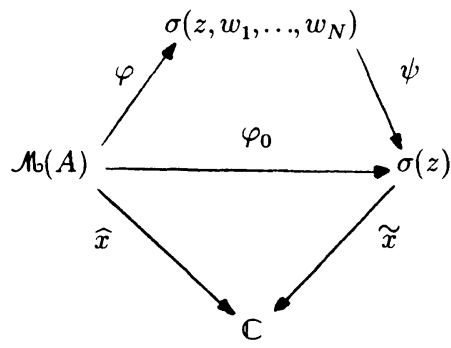

Let $\widetilde{A}=\{\widetilde{x}: x \in A\}$, equipped with the compact-open topology. We first show that $\widetilde{A} \subseteq H(\sigma(z))$.

Let $x \in A$, and let $P_{k}$ be a sequence of polynomials in $N+1$ variables such that $P_{k}\left(z, w_{1}, \ldots, w_{N}\right)$ converges to $x$. Define $r_{k}(t)$ to be the rational function $P_{k}\left(t,\left(t-\lambda_{1}\right)^{-1}, \ldots,\left(t-\lambda_{N}\right)^{-1}\right)$. Then

$$
f_{t}\left(P_{k}\left(z, w_{1}, \ldots, w_{N}\right)\right)=P_{k}\left(f_{t}(z), f_{t}\left(w_{1}\right), \ldots, f_{t}\left(w_{N}\right)\right)=r_{k}(t)
$$

and so $r_{k}(t)$ converges to $f_{t}(x)=\widetilde{x}(t)$. This means that $\widetilde{x}$ is the pointwise limit of rational functions on $\sigma(z)$, whose poles occur at $\lambda_{1}, \ldots, \lambda_{N}$. (Note that each $\lambda_{1}$ lies outside $\sigma(z)$, since $z-\lambda_{\imath} e$ is invertible.) We now show that the convergence $r_{k}(t) \rightarrow \widetilde{x}(t)$ is uniform on compact sets. This will prove that $\widetilde{x}$ is analytic on $\sigma(z)$.

First note that since $A$ has no topological divisors of zero, for each $n \in \mathbb{N}$ there exists $m>n$ such that $M_{n} \subset$ int $M_{m}$ (see Arens [13]). So, without loss of generality (by replacing the original sequence of seminorms by an equivalent one) we may assume that $M_{n} \subset \operatorname{int} M_{n+1} \subset M_{n+1}(n=1,2, \ldots)$. Now $\varphi_{0} \mid M_{n}$ is a homeomorphism onto its image (because $\varphi \mid M_{n}$ is, and $\psi$ is a bijection). Thus it follows that $\varphi_{0}\left(M_{n}\right) \subset \operatorname{int} \varphi_{0}\left(M_{n+1}\right) \subset \varphi_{0}\left(M_{n+1}\right)$, $n=1,2, \ldots$, and $\sigma(z)=\bigcup \varphi_{0}\left(M_{n}\right)=\bigcup \operatorname{int} \varphi_{0}\left(M_{n}\right)$.

Now let $S$ be a compact subset of $\sigma(z)$. By the preceding paragraph, there exists $n \in \mathbb{N}$ 
such that $S \subset \operatorname{int} \varphi_{0}\left(M_{n}\right)$, so that $\varphi_{0}^{-1}(S) \subset M_{n}$. Thus $\varphi_{0}^{-1}(S)$ is a compact (and hence equicontinuous) subset of $\mathcal{M}(A)$. Now $P_{k} \rightarrow x$, so the continuity of the Gelfand nuap implies that $\widehat{P}_{k} \rightarrow \widehat{x}$ in $\widehat{A}$, i.e., uniformly on compact subsets of $\mathcal{M}(A)$. Thus for $\epsilon>0$ and sufficiently large $k,\left|\widehat{P}_{k}\left(f_{t}\right)-\widehat{x}\left(f_{t}\right)\right|<\epsilon$ for $f_{t} \in \varphi_{0}^{-1}(S)$, which is the same as $\left|r_{k}(t)-\widetilde{x}(t)\right|<\epsilon$ for $t \in S$. This shows that each $\widetilde{x}$ is the limit of rational functions whose poles lie outside $\sigma(z)$; the convergence is uniform on compact subsets of $\sigma(z)$, and hence $\widetilde{x}$ is analytic on $\sigma(z)$. It follows that $\widetilde{A} \subseteq H(\sigma(z))$.

Now if $h \in H(\sigma(z))$, then by the functional calculus for $F$-algebras there exists $y \in A$ such that $\widehat{y}(f)=h(\widehat{z}(f))$ for $f \in \mathcal{M}(A)$. Therefore $h=\widetilde{y}$ and so $\widetilde{A}=H(\sigma(z))$. This last equality shows that the polynomials in $t,\left(t-\lambda_{1}\right)^{-1}, \ldots,\left(t-\lambda_{N}\right)^{-1}$ (i.e. the rational functions with poles at $\left.\lambda_{1}, \ldots, \lambda_{N}\right)$ are dense in $H(\sigma(z))$. Runge's Theorem [11, page 200] implies that $\sigma(z)$ is a finitely connected domain.

THEOREM 2.2. The algebra $A$ is a Runge algebra with no topological divisors of zero if and only if $A$ is usomorphic to $H(\Omega)$ for some finitely connected domain $\Omega$.

ProOF. That $H(\Omega)$ has the indicated properties is discussed in the first paragraph of this section. Conversely, suppose $A$ has no topological divisors of zero and is generated by elements $\left(z-\lambda_{1} e\right)^{-1}, \ldots,\left(z-\lambda_{N} e\right)^{-1}$, where $\lambda_{1}, \ldots, \lambda_{N} \in \mathbb{C}$. The map $\varphi_{0}: \mathcal{M}(A) \rightarrow \sigma(z)$ defined in the proof of Proposition 2.1 is a homeomorphism, because the topology on $\mathcal{M}(A)$ is the weak topology generated by $\widehat{A}$, and $\widehat{x} \circ \varphi_{0}^{-1}=\widetilde{x}$ is continuous for each $\widehat{x} \in \widehat{A}$. Let $G: \widehat{A} \rightarrow \widetilde{A}$ be the $\operatorname{map} G(\widehat{x})=\widetilde{x}$. We claim that

$$
A \stackrel{\Gamma}{\longrightarrow} \widehat{A} \stackrel{G}{\longrightarrow} \widetilde{A}
$$

is an isomorphism. Since $G(\widehat{x})=\widehat{x} \circ \varphi_{0}^{-1}, G$ is an isomorphism, and since $A$ is semisimple, $\Gamma$ is a bijection. Thus $G \circ \Gamma$ is a continuous bijection onto the $F$-algebra $\widetilde{A}$, hence is open by the open mapping theorem, and so $A \simeq \widetilde{A}$. But, as in Proposition 2.1, $\widetilde{A}=H(\sigma(z))$ and $\sigma(z)$ is finitely connected. This completes the proof.

If each of the seminorms $p_{k}$ of an $F$-algebra $A$ satisfies $p_{k}\left(x^{2}\right)=p_{k}(x)^{2}$ for all $x \in A$, then $A$ is a uniform algebra. For such an algebra, $p_{k}(x)=\sup \left\{|\widehat{x}(f)|: f \in M_{k}\right\}$ for all $x \in A, k \in \mathbb{N}$. Thus the Gelfand map $\Gamma$ is a homeomorphism of $A$ onto a complete subalgebra $\widehat{A}$ of $C(\mathcal{H}(A))$. A derivation on an $F$-algebra $A$ is a linear transformation $D: A \rightarrow A$ satisfying $D(x y)=x D(y)+D(x) y$. Carpenter [5] used the existence of a derivation on a uniform $F$ algebra to characterize $H(\Omega)$. His result provides one of the equivalences in the following theorem.

THEOREM 2.3. Let $A$ be a uniform Runge algebra which is generated by the elements $z,\left(z-\lambda_{1} e\right)^{-1}, \ldots,\left(z-\lambda_{N} e\right)^{-1}$, where $\lambda_{1}, \ldots, \lambda_{N} \in \mathbb{C}$. The following are equivalent conditions on $A$.

(a) A has no topological divisors of zero.

(b) The spectrum $\sigma(z)$ is an open subset of $\mathbb{C}$.

(c) A has a derivation $D$ such that $D(z)=e$.

(d) A us isomorphic to $H(\Omega)$ for a finitely connected domain $\Omega$. 
Proof. Clearly (d) implies (a), (b), and (c). That (a) implies (d) follows from Theorem 2.2, and that (c) implies (d) follows from Carpenter [5]. We prove that (b) implies (d). Let $w_{\imath}=\left(z-\lambda_{\imath} \epsilon\right)^{-1}, \imath=1, \ldots, N$. Observe first that $\sigma\left(z, w_{1}, \ldots, w_{N}\right)$ is homeomorphic to $\sigma(z)$, and $\sigma_{n}\left(z, w_{1}, \ldots, w_{N}\right)$ to $\sigma_{n}(z)$, via the map $\psi$ defined in the proof of Proposition 2.1. We show that $\left\{\sigma_{n}\left(z, w_{1}, \ldots, w_{N}\right)\right\}$ is a $k$-covering sequence for $\sigma\left(z, w_{1}, \ldots, w_{N}\right)$ by showing that $\left\{\sigma_{n}(z)\right\}$ is a $k$ covering sequence for $\sigma(z)$ ( $k$-covering means that the sequence covers every compact subset). But $\sigma(z)$ is the increasing union of the $\sigma_{n}(z)$ and is open, so $\sigma(z)=\bigcup \operatorname{int} \sigma_{n}(z)$. Thus \{int $\left.\sigma_{n}(z)\right\}$ is an open cover for any compact subset $K$ of $\sigma(z)$, so $K \subseteq \sigma_{n}(z)$ for some $n \in \mathbb{N}$. This proves that $\left\{\sigma_{n}\left(z, w_{1}, \ldots, w_{N}\right)\right\}$ is a $k$-covering for $\sigma\left(z, w_{1}, \ldots, w_{N}\right)$, and so by [7, Theorem 1.3] the map $\varphi: \mathcal{H}(A) \rightarrow \sigma\left(z, w_{1}, \ldots, w_{N}\right)$ is a homeomorphism. Thus $\mathcal{H}(A)$ is homeomorphic to $\sigma(z)$ by the map $\varphi_{0}: f \mapsto f(z)$. This, together with the fact that $A$ is a uniform algebra, allows us to consider $\widehat{A}$ (and hence $A$ ) as an algebra of continuous functions on $\sigma(z)$ with the compact-open topology. Under this identification, for $t \in \sigma(z)$ we have $\widehat{z}(t) \equiv \widehat{z}\left(\varphi_{0}^{-1}(t)\right)=\left[\varphi_{0}^{-1}(t)\right](z)=t, \quad$ and similarly $\widehat{w}_{1}(t)=\left(t-\lambda_{1}\right)^{-1}, \ldots, \quad \widehat{w}_{N}(t)=\left(t-\lambda_{N}\right)^{-1}$; moreover, these functions generate $\widehat{A}$. By Runge's theorem [11, page 200], $\widehat{A}$ (and hence $A$ ) is isomorphic to $H(\Omega)$ for a finitely connected domain $\Omega$.

\section{BASES GENERATED BY $z$ AND $z^{-1}$}

In this section we assume that $A$ is an $F$-algebra with a basis generated by $z$ and $z^{-1}$; that is, for each $x \in A$, there is a unique sequence $\left\{\alpha_{n}: n \in \mathbb{Z}\right\}$ of scalars such that

$$
x=\sum_{n=-\infty}^{\infty} \alpha_{n} z^{n}
$$

where the series converges independently in the positive and negative directions of summation. (In [14] algebras with bases of the form $\left\{z^{n}: n \in \mathbb{N}\right\}$ are studied.) We first analyze the spectrum of $z$ for such algebras.

LEMMA 3.1. Let $A$ be an F-algebra with a basis $\left\{z^{n}: n \in \mathbb{Z}\right\}$, and let $r=1 / \rho\left(z^{-1}\right), R=\rho(z)$, where $\rho$ denotes the spectral radius. If $\rho(z) \rho\left(z^{-1}\right) \neq 1$, let $S=\{\lambda \in \mathbb{C}: r<|\lambda|<R\}$, and if $\rho(z) \rho\left(z^{-1}\right)=1$, let $S=\{\lambda \in \mathbb{C}:|\lambda|=R\}$. Then $S \subset \sigma(z) \subset \bar{S}$.

ProOF. If $z-\lambda e$ is invertible, $(z-\lambda e)^{-1}=\sum_{n=-\infty}^{\infty} \alpha_{n} z^{n}$. Then

$$
\begin{aligned}
e & =(z-\lambda e) \sum_{n=-\infty}^{\infty} \alpha_{n} z^{n}=\sum_{n=-\infty}^{\infty} \alpha_{n} z^{n+1}-\sum_{n=-\infty}^{\infty} \lambda \alpha_{n} z^{n} \\
& =\sum_{n=-\infty}^{\infty}\left(\alpha_{n-1}-\lambda \alpha_{n}\right) z^{n} .
\end{aligned}
$$

Thus $\alpha_{-1}-\lambda \alpha_{0}=1$ and $\alpha_{n-1}-\lambda \alpha_{n}=0$ for $n \neq 0$. When $\lambda \neq 0$, we can write the coefficients in terms of $\alpha_{0}$ to get $\alpha_{n}=\alpha_{0} / \lambda^{n}, n \geq 0$, and $\alpha_{-n}=\alpha_{0} \lambda^{n}+\lambda^{n-1}, n \geq 1$. Thus

$$
(z-\lambda e)^{-1}=\alpha_{0} \sum_{n=0}^{\infty}\left(\frac{z}{\lambda}\right)^{n}+\sum_{n=1}^{\infty}\left(\alpha_{0} \lambda^{n}+\lambda^{n-1}\right) z^{-n}
$$

Now suppose $\lambda \notin \sigma(z)$, with $\lambda \neq 0$. Then $z-\lambda e$ is invertible and its inverse is given by the series (3.1). Let $f \in \mathcal{M}(A)$. We consider two cases. If $\alpha_{0} \neq 0$ in (3.1), then the series $\sum_{n=0}^{\infty}(f(z) / \lambda)^{n}$ converges in $\mathbb{C}$, and so $|f(z) / \lambda|<1$. If follows that $|f(z)|<|\lambda|$ for all 
$f \in \mathcal{M}(A)$ and hence $|\lambda| \geq \rho(z)$. If $\alpha_{0}=0$ in $(3.1)$, then the series $\lambda^{-1} \sum_{n=1}^{\infty}\left(f\left(z^{-1}\right) \lambda\right)^{n}$ converges, so that $\left|f\left(z^{-1}\right) \lambda\right|<1$. Thus $|\lambda|<1 /\left|f\left(z^{-1}\right)\right|$ for all $f \in \mathcal{M}(A)$ and so $|\lambda| \leq 1 / \rho\left(z^{-1}\right)$. Since 0 belongs to neither $S$ nor $\sigma(z)$, this shows that $S \subset \sigma(z) \subset \bar{S}$.

TIIEOREM 3.2. Let $A$ be an F-algebra with a basıs $\left\{z^{n}: n \in \mathbb{Z}\right\}$. Then $A$ is semisimple and its topology is given by an increasing sequence of norms.

Proof. Suppose $x \in \operatorname{Rad}(A), x=\sum_{-\infty}^{\infty} \alpha_{n} z^{n}$. Then $f(x)=\sum_{-\infty}^{\infty} \alpha_{n} f(z)^{n}=0$ for all $f \in \mathcal{M}(A)$. Thus the Laurent series $\sum_{-\infty}^{\infty} \alpha_{n} t^{n}$ converges to 0 for $t \in \sigma(z)$, and since $\sigma(z)$ has a limit point (Lemma 3.1), $\alpha_{n}=0$ for all $n$. Thus $x=0$ and $A$ is semisimple.

Let $K$ be an infinite compact subset of $\mathcal{M}(A)$. (Such a set must exist, for otherwise $\mathcal{M}(A)$ being hemicompact $[10$, page 22] would be countable, contradicting Lemma 3.1 and the fact that $\varphi$ is a bijection.) Since $K$ is equicontinuous there exists $c>0$ and a seminorm $p_{k}$ such that $|f(x)| \leq c p_{k}(x)$ for all $f \in K, x \in A$. Thus $p_{k}(x)=0$ implies $f(x)=0$ for all $f \in K$, and since $\varphi(K)$ has a limit point in $\mathbb{C}$, an argument as in the preceding paragraph shows that $x=0$. This means that $p_{k}$ is a norm, and hence so is $p_{n}$ for $n \geq k$.

THEOREM 3.3. Let $A$ be a Banach algebra with a basis $\left\{z^{n}: n \in \mathbb{Z}\right\}$. Then $\mathcal{M}(A)$ is homeomorphic to $\bar{\Omega}=\left\{\lambda \in \mathbb{C}: 1 / \rho\left(z^{-1}\right) \leq|\lambda| \leq \rho(z)\right\}$.

Proof. Since $A$ is a Banach algebra, $\mathcal{H}(A)$ is homeomorphic to $\sigma\left(z, z^{-1}\right)$, which in turn is homeomorphic to $\sigma(z)$ via the projection map $\pi_{1}: \sigma\left(z, z^{-1}\right) \rightarrow \sigma(z)$ which takes $\left(f(z), f\left(z^{-1}\right)\right)$ to $f(z) . \quad\left(\pi_{1}\right.$ is one-to-one because each $f \in \mathcal{H}(A)$ is completely determined by its value at $z$.) Since $\sigma(z)$ is compact, it is equal to $\bar{\Omega}$ by Lemma 3.1 .

An example of a Banach algebra with a basis of the type being considered is $\ell^{1}(\mathbf{Z})$. We now construct another example. If $f(t)=\sum_{-\infty}^{\infty} a_{n} t^{n}$ is analytic on the annulus $\operatorname{Ann}(0 ; r, R)=\{t \in \mathbb{C}: r<|t|<R\}$, and if $r<s<R$, let $\|f\|_{s}=\sum_{-\infty}^{\infty}\left|a_{n}\right| s^{n}$. The limits $\lim _{s \rightarrow r^{+}}\|f\|_{s}=\|f\|_{r}$ and $\lim _{s \rightarrow R^{-}}\|f\|_{s}=\|f\|_{R}$ both exist. Let $A(r, R)$ be the space of such functions for which the norm

$$
\|f\|=\max \left\{\|f\|_{r},\|f\|_{R}\right\}
$$

is finite. By an argument as in [15], $A(r, R)$ is a Banach algebra with basis $\left\{z^{n}: n \in \mathbf{Z}\right\}$, where $z$ is the function $z(t)=t$. Abel's theorem shows that $A(r, R)$ consists of those analytic functions $f(t)=\sum_{-\infty}^{\infty} a_{n} t^{n}$ on $\operatorname{Ann}(0 ; r, R)$ for which

$$
\sum_{-\infty}^{\infty}\left|a_{n}\right| r^{n}<\infty \text { and } \sum_{-\infty}^{\infty}\left|a_{n}\right| R^{n}
$$

We show that this example is typical of Banach algebras with bases of the type under consideration.

THEOREM 3.4. Let $A$ be a Banach algebra with an unconditional basis $\left\{z^{n}: n \in \mathbb{Z}\right\}$. If $\rho(z)=\|z\|$ and $\rho\left(z^{-1}\right)=\left\|z^{-1}\right\|$, then $A$ is isomorphic to $A(r, R)$, where $r=1 / \rho\left(z^{-1}\right)$ and $R=\rho(z)$. 
ProOf. For $x \in A$ we define $\widetilde{x}$ as in the proof of Proposition 2.1 ; that is, $\widetilde{x}: \sigma(z) \rightarrow \mathbb{C}$ is given by $\widetilde{x}(t)=\widehat{x}\left(\varphi_{0}^{-1}(t)\right)$. For $x=\sum_{-\infty}^{\infty} \alpha_{n} z^{n}$, we have $\widetilde{x}(t)=\sum_{-\infty}^{\infty} \alpha_{n} t^{n}$, and since the basis is unconditional, each $\widetilde{x}(t)$ is an absolutely convergent series for every $t \in \sigma(z)$. Since $\sigma(z)$ is compact, we have by Theorem 3.3 that $r, R \in \sigma(z)$ and so we conclude from (3.2) that $\tilde{x} \in A(r, R)$. Thus we can define the map

$$
\begin{aligned}
L: A & \rightarrow A(r, R) \\
: x & \mapsto \widetilde{x}
\end{aligned}
$$

Clearly $L$ is one-to-one, linear, and multiplicative. If $f \in A(r, R)$, then there is a sequence $\left\{\beta_{n}\right\}$ of complex numbers such that $f(t)=\sum_{-\infty}^{\infty} \beta_{n} t^{n}$ and this series converges for $t \in \sigma(z)$ by Theorem 3.3. But $\rho(z) \in \sigma(z)$, so $\sum_{-\infty}^{\infty}\left|\beta_{n}\right| \rho(z)^{n}<\infty$, and hence by assumption $\sum_{n=0}^{\infty}\left|\beta_{n}\right|\left\|z^{n}\right\|<\infty$. Similarly, $1 / \rho\left(z^{-1}\right) \in \sigma(z)$, so $\sum_{-\infty}^{\infty}\left|\beta_{n}\right| \rho\left(z^{-1}\right)^{-n}=\sum_{-\infty}^{\infty}\left|\beta_{-n}\right| \rho\left(z^{-1}\right)^{n}$ $<\infty$, and hence $\sum_{n=0}^{\infty}\left|\beta_{-n}\right| \rho\left(z^{-n}\right)<\infty$. These two facts show that the series $\sum_{-\infty}^{\infty} \beta_{n} z^{n}$ converges in $A$, to $y$, say. Clearly $\widetilde{y}=f$ and hence $L$ is onto. Thus $L$ is an algebraic isomorphism from $A$ onto $A(r, R)$ and, since $A(r, R)$ is semisimple, $L$ is continuous. The open mapping theorem now shows that $L$ is also a topological isomorphism.

\section{REFERENCES}

1. RUDIN, W. Analyticity, and the maximum modulus principle, Duke Math. $\underline{\text { J. }} \underline{20}$ (1953), 449-458.

2. BIRTEL, F. T. Singly-generated Liouville F-algebras, Mich. Math. J. 11 (1964), 84-94.

3. WANG, D. and WATSON, S. A characterization of the algebra of holomorphic functions on a simply connected domain, Internat. J. Math. \& Math. Sci. 12 (1989), 65-68.

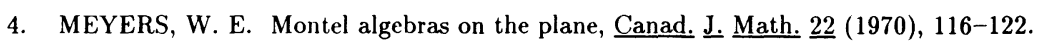

5. CARPENTER, R. Characterizing hol $(\Omega)$, Proc. Amer. Math. Soc. $\underline{53}$ (1975), 57-59.

6. ARENS, R. F. Dense inverse limit rings, Mich. Math. J. $\underline{5}$ (1958), 169-182.

7. BROOKS, R. M. On the spectrum of finitely-generated locally $m$-convex algebras, $\underline{\text { Studia }} \underline{\text { Math. }} \underline{29}$ (1958), 143-150.

8. HEAL, E. R. and WINDHAM, M. P. Finitely generated $F$-algebras with applications to Stein manifolds, Pacific J. Math. $\underline{51}$ (1974), 459-465.

9. STEWART, J. and WATSON, S. Topological algebras with finitely-generated bases, Math. Annalen $\underline{271}$ (1985), 315-318.

10. MICHAEL, E. A. Locally multiplicatively-convex topological algebras, Mem. Amer. Math. $\underline{\text { Soc. }} \underline{11}$, (1952).

11. CONWAY, J. B. Functions of One Complex Variable, Second Edition, Springer-Verlag, New York, 1978.

12. ŻELAZKO, W. Selected Topics in Topological Algebras, Aarhus Universitet Lecture Notes Series No. $31,1971$.

13. ARENS, R. F. Joint spectra in topological algebras, Conference on Analytic Functions, Inst. for Adv. Study, Vol. 2, Princeton Univ. Press, 1957.

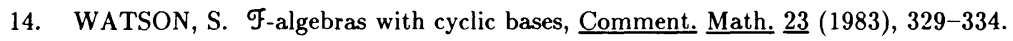

15. ARSOVE, M. G. Proper bases and homomorphisms in spaces of analytic functions, Math. Annalen $\underline{135}$ (1958), 235-243. 


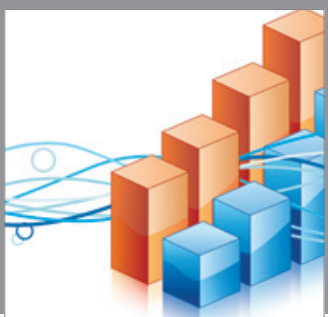

Advances in

Operations Research

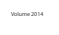

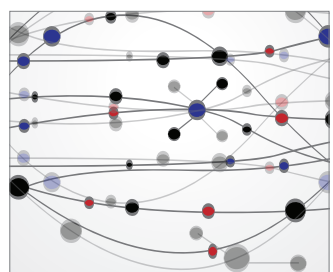

\section{The Scientific} World Journal
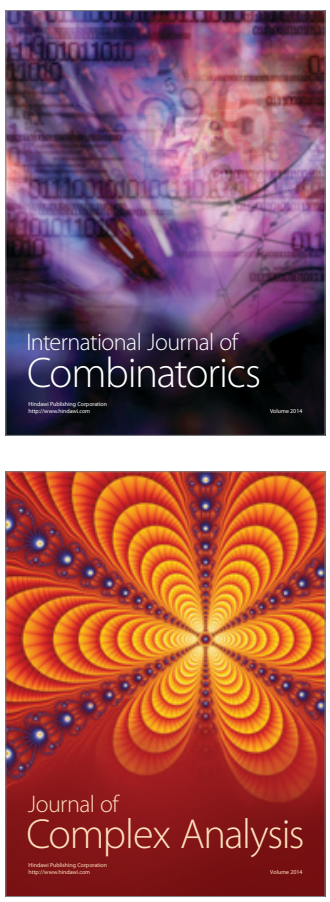

International Journal of

Mathematics and

Mathematical

Sciences
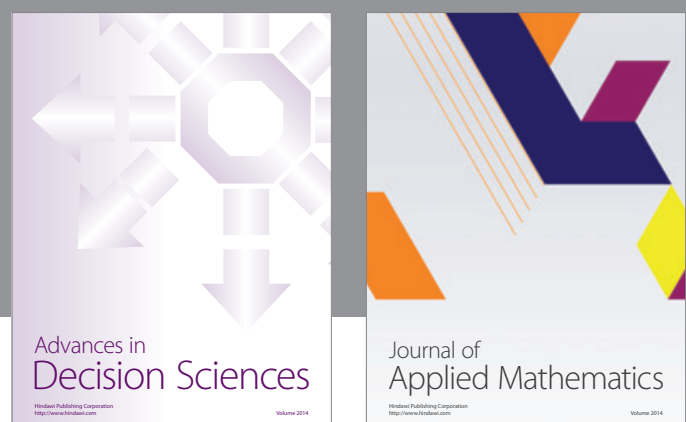

Journal of

Applied Mathematics
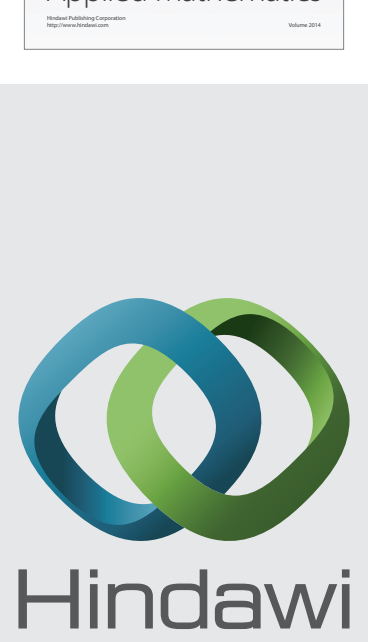

Submit your manuscripts at http://www.hindawi.com
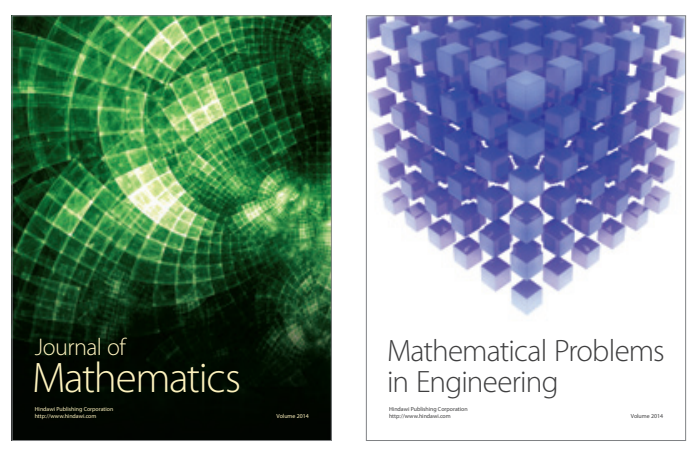

Mathematical Problems in Engineering
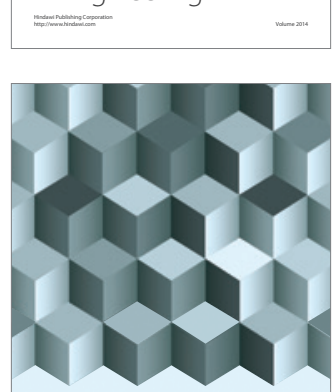

Journal of

Function Spaces
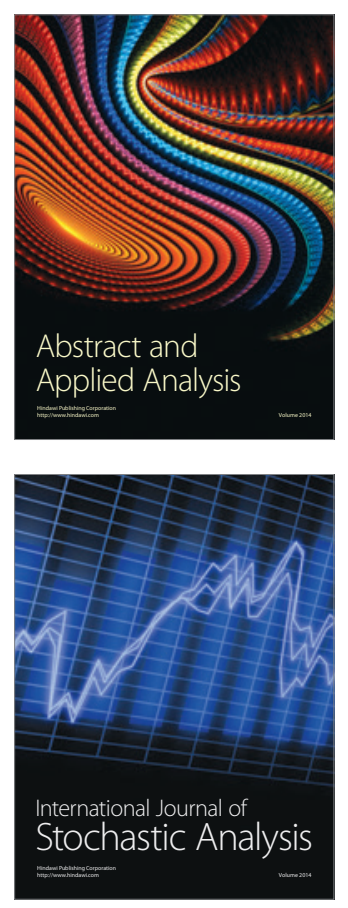

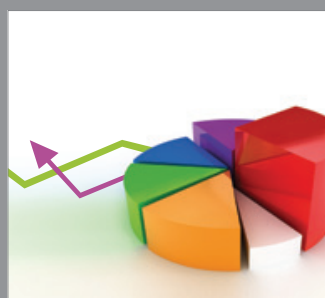

ournal of

Probability and Statistics

Promensencen
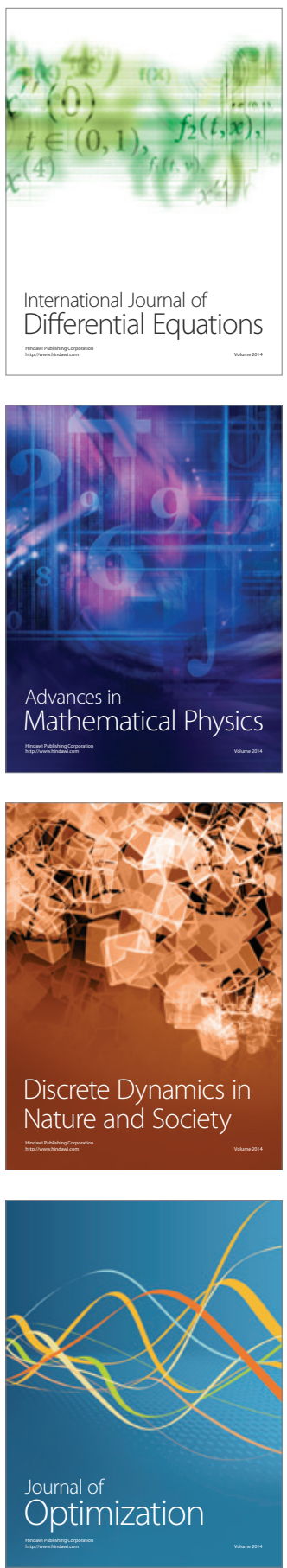